\title{
On-line detection of voltage transient disturbances using ANNs
}

\author{
F. J. Alcántara, J. R. Vázquez, P. Salmerón, S. P. Litrán, M. I. Arteaga Orozco \\ Departamento de Ingeniería Eléctrica y Térmica \\ Escuela Politécnica Superior. Universidad de Huelva \\ Crta. Palos de la Frontera s/n., 21819, Palos de la Frontera, Huelva, Spain \\ Phone: +34-959217579, Fax: +34-959217304 \\ e-mail: benju@uhu.es, vazquez@uhu.es, patricio@uhu.es, salvador@uhu.es, maria.arteaga@die.uhu.es
}

\begin{abstract}
The non-quality phenomena of the supply voltage in electrical power systems include transient disturbances as frequency variations, sags, swells, flicker or interruptions. In this work, a method to detect and measure some transient disturbances based on Artificial Neural networks (ANNs) will be presented. A Feedforward network has been trained to detect the initial time, the final time and the magnitude of a voltage disturbance. The design and training process of an ANN specialized in voltage sags detection will be presented. The performance of the designed measure method will be tested in a simulation platform designed in Matlab/Simulink through the analysis of a practical case.
\end{abstract}

\section{Keywords}

Electrical power quality, transient disturbances, measurement, artificial neural networks, feedforward

\section{Introduction}

Nowadays, the modern electrical equipment needs a high quality supply voltage. Problems appear with voltage disturbances as frequency variations, sags or dips, swells, flicker or voltage interruptions, [1-3]. For a suitable detection of the different disturbances, the adequate measurement method must be chosen.

The artificial neural networks had been applied successfully in several topics of the Electrical Engineering, including the detection of some voltage disturbances, [4-5]. The speed and the parallelism of the calculations are the main advantages of these techniques. So, the neural networks applied to the voltage disturbance detection can be competitive with respect to other techniques as, for example, the based on the Wavelet Transform, [6].

In this work, a Feedforward ANN has been designed for transient disturbance measurements, specifically, for voltage sags detection. To probe the performance of designed measurement system, a network has been trained and simulated in a Matlab/Simulink platform. After an off-line training process using a Backpropagation algorithm, the network was connected to an electrical system for the on-line detection of supply voltage sags. The results of a practical case will verify the suitable performance of the designed network.

\section{Neural network architecture}

The Artificial Neural Networks includes a large number of strongly connected elements: the artificial neurons, a biological neuron abstraction. The model of an artificial neuron in a schematic configuration is shown in figure 1

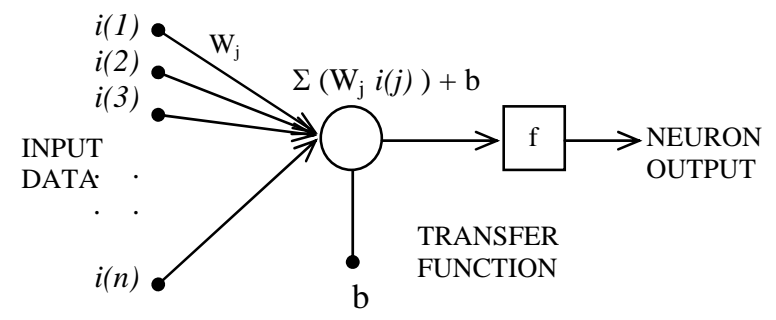

Fig. 1. Artificial Neuron Model

The input data $i(1), i(2), i(3), \ldots, i(n)$ flow through the synapses weights $\mathrm{W}_{\mathrm{j}}$. These weights amplify or attenuate the inputs signals before the addition at the node represented by a circle. The summed data flows to the output through a transfer function, $\mathrm{f}$, which may be the threshold one, the sign one, the linear threshold one or the pure linear one. Otherwise, it may be a continuous non-linear function like the sigmoid one, the inverse tan one, the hyperbolic one or the gaussian one, according the application.

The neurons are interconnected creating different layers. The feedforward architecture is the most commonly adopted. The scheme is shown in figure 2 . 


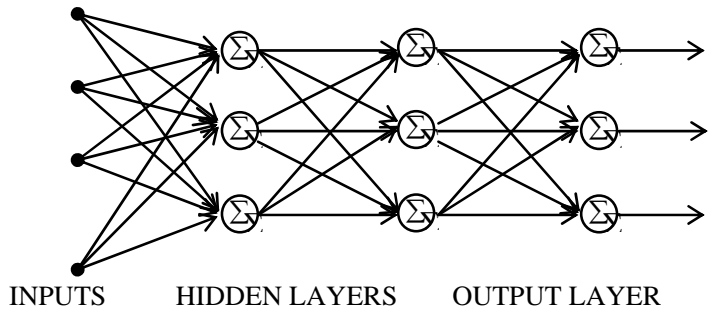

Fig. 2. Feedforward Neural Network Architecture

The feedforward ANN scheme includes the inputs, some hidden layers, an output layer and the outputs. In figure 2 , the circles represent neurons. The number of output layer neurons is equal to the number of outputs. Thus, the feedforward architecture computes the input data in parallel way, faster than the sequential algorithm of the computers.

This network can be trained to give a desired pattern at the output, when the corresponding input data set is applied. This training process is carried out with a large number of input and output target data. These data can be obtained using a simulation platform or an experimental system. The training method most commonly used is the backpropagation algorithm. The network is initially untrained and is initialized with random weights.

The backpropagation training process consists of two phases: in the first one an input is presented to the network, that is propagated to the output. In each neuron of different layers is calculated.

$$
\hat{y}(k)=f\left[\sum_{j}\left(W_{j} \cdot i(j)\right)+b\right]=f(v)
$$

In equation $1, v$ is the activation potential of the neuron and $\hat{y}(k)$ represents the neuron output at the iteration $\mathrm{k}$. Thus, the network output $\hat{O}$ is obtained at the output layer.

In the second phase, the error between the desired output of the network and the real output, $e(k)$, is propagated to the back, adapting the network weights to minimize the addition of square errors in the output layer, $\varepsilon(k)$.

$$
e(k)=o(k)-\hat{o}(k), \quad \varepsilon(k)=\sum_{j \in O} e_{j}^{2}(k)
$$

Where $o(k)$ is the desired output and $O$ is the set of output neurons. In this phase, the neuron outputs remain constant, and the weights are changed according the following formula.

$$
\Delta W_{i j}(k)=\eta \delta_{j}(k) y_{i}(k)
$$

$W_{i j}$ represents the weight between the neurons $\mathrm{i}$ and $\mathrm{j}, \eta$ is the learning rate, $y_{i}(k)$ are neuron inputs that coincides with outputs of the preceding layer neurons, and $\delta_{j}(k)$ is the local gradient of the neuron, that is defined as

$$
\Delta W_{i j}(k)=-\frac{\partial \varepsilon(k)}{\partial v_{j}(k)}
$$

The initial output pattern is compared with the desired output pattern and the weights are adjusted by the algorithm to minimize the error $\varepsilon(k)$. The iterative process finishes when the error becomes near null.

In this work, the neural network has been designed with three inputs, corresponding to the voltage at three consecutive time instants $v(t), v(t-\Delta t)$ and $v(t-2 \Delta t)$, two hidden layers of 20 and 12 neurons with sigmoid transfer function, and an output layer with only one neuron with pure linear transfer function.

\section{Simulation platform}

To simulate the proposed measurement system, including the ANN training process and its on-line performance, a Matlab/Simulink platform has been designed.

To carry out the network training process, a set of input voltage waveforms was generated. Voltage sags of length and depth known (80\% in the example of figure 3 ) were added to the sinusoidal voltage waveform. The input data of the training were completed with voltage sags of several lengths and several depths. It was necessary, not only to include samples of several magnitude sags in the training data set, but also voltage sags of different initial and final instants, $\mathrm{t}_{0} \mathrm{y} \mathrm{t}_{1}$, to train correctly the network, and it can respond better to the presence of sags that can begin and finish at any time instant between a signal period.

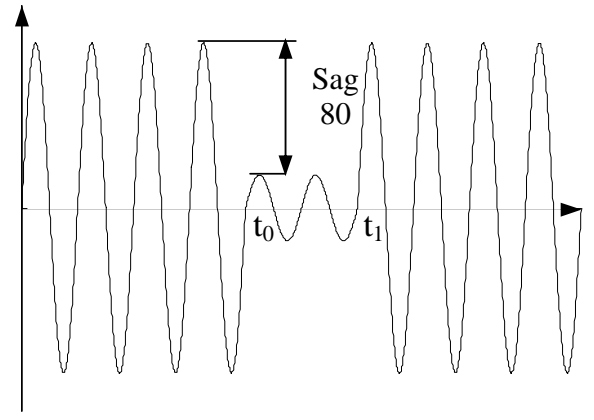

Fig. 3. Example of voltage sag

Figure 4 shows the Simulink diagram used to generate the network inputs.

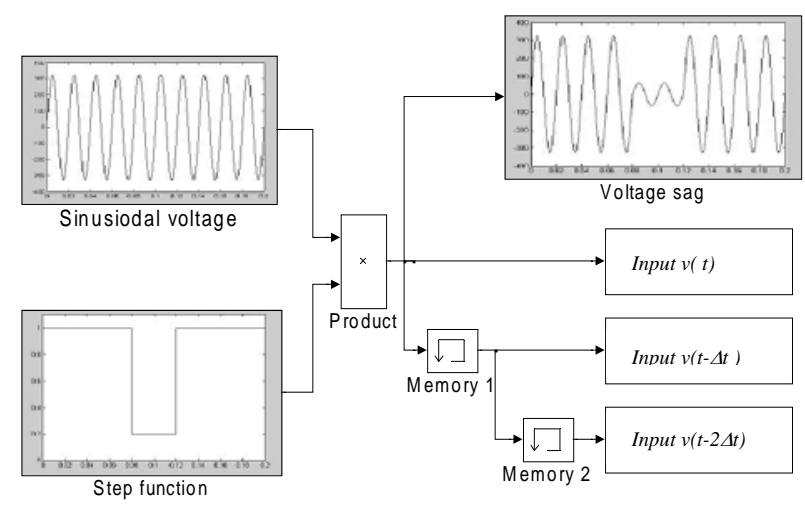

Fig. 4. Simulink diagram of input data generation 
An incremental algorithm was applied to train the neural network, in which three inputs data are considered in each time step, corresponding to $v(t), v(t-\Delta t)$ and $v(t-2 \Delta t)$. $\Delta t$ is the time step between two consecutive instants. The relative voltage amplitude was considered as desired network output. So, the output will be $o=1$ if the voltage has the nominal value and $o=0,2$ when the voltage sag has a $80 \%$ depth.

The training process of the neural network was carried out helped by the Neural Network Matlab toolbox, using the trainlm function. Different network topologies were issues. Figure 5 shows the error evolution during the training process using an ANN with 8, 4 and 1 neurons and figure 6 using an ANN with 20, 12 and 1 neurons.

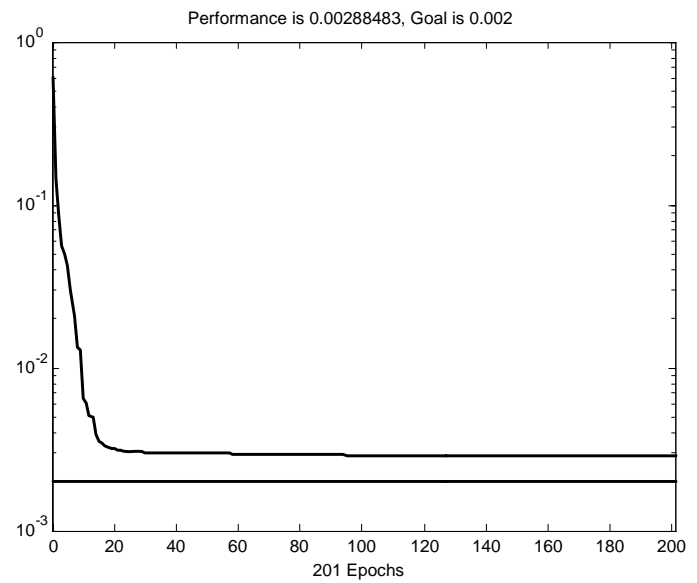

Fig. 5. Error evolution during the training process of neural network with 8, 4 and 1 neurons.

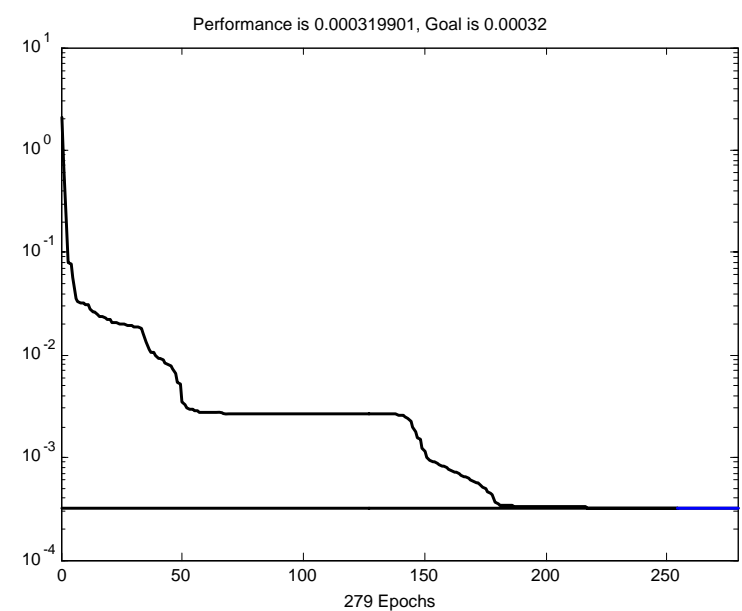

Fig. 6. Error evolution during the training process of neural network with 20, 12 and 1 neurons.

In the first case the maximum error didn't became small, and in the second case the maximum error was equal to 0,00032. In this work, the second network has been choose.

\section{Results of practical cases}

\section{A. Detection of ideal sags}

After the network training process, the measurement system performance was tested in the presence of three voltage sags of $69 \%, 44 \%$ and $18 \%$ which happens in different time instants. The voltage waveform is shown in figure 7 , and the results of the designed measurement system are shown in figure 8 .

a)
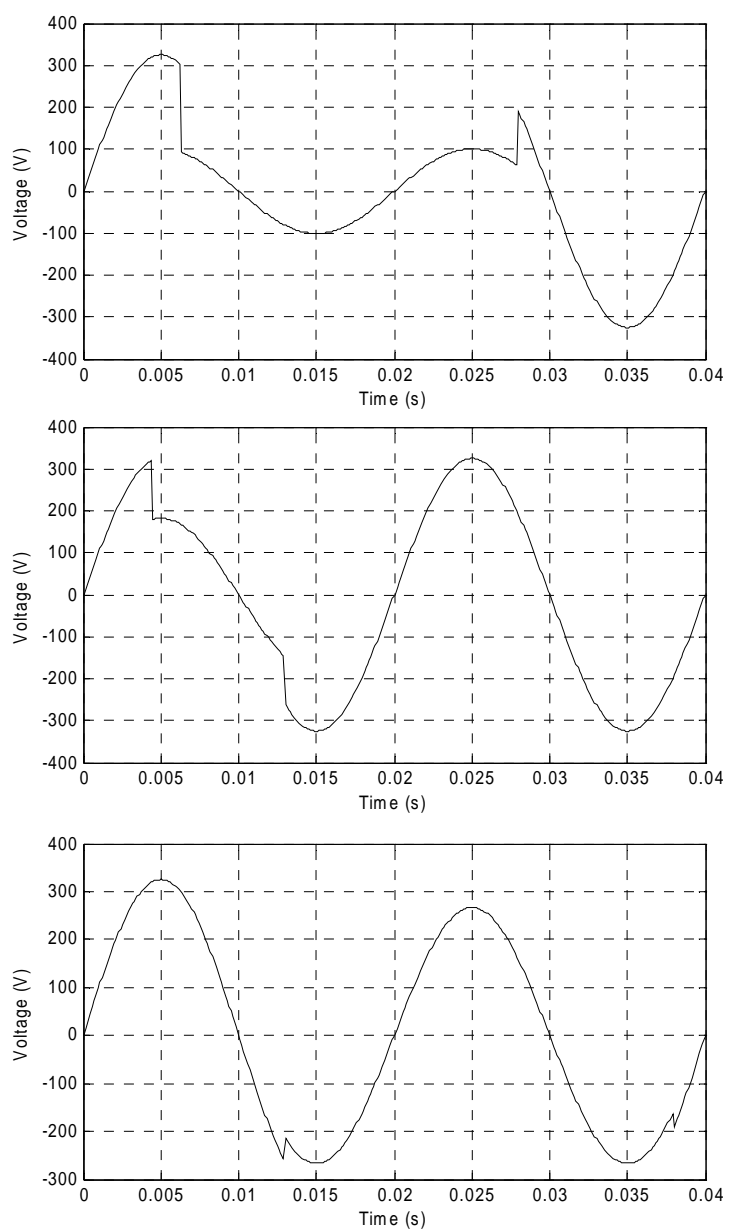

Fig. 7. Voltage waveforms of the three voltage sags

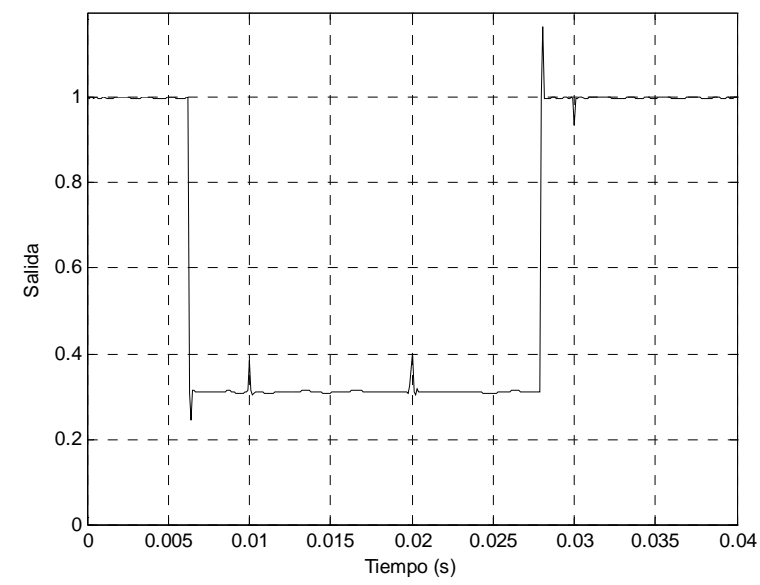



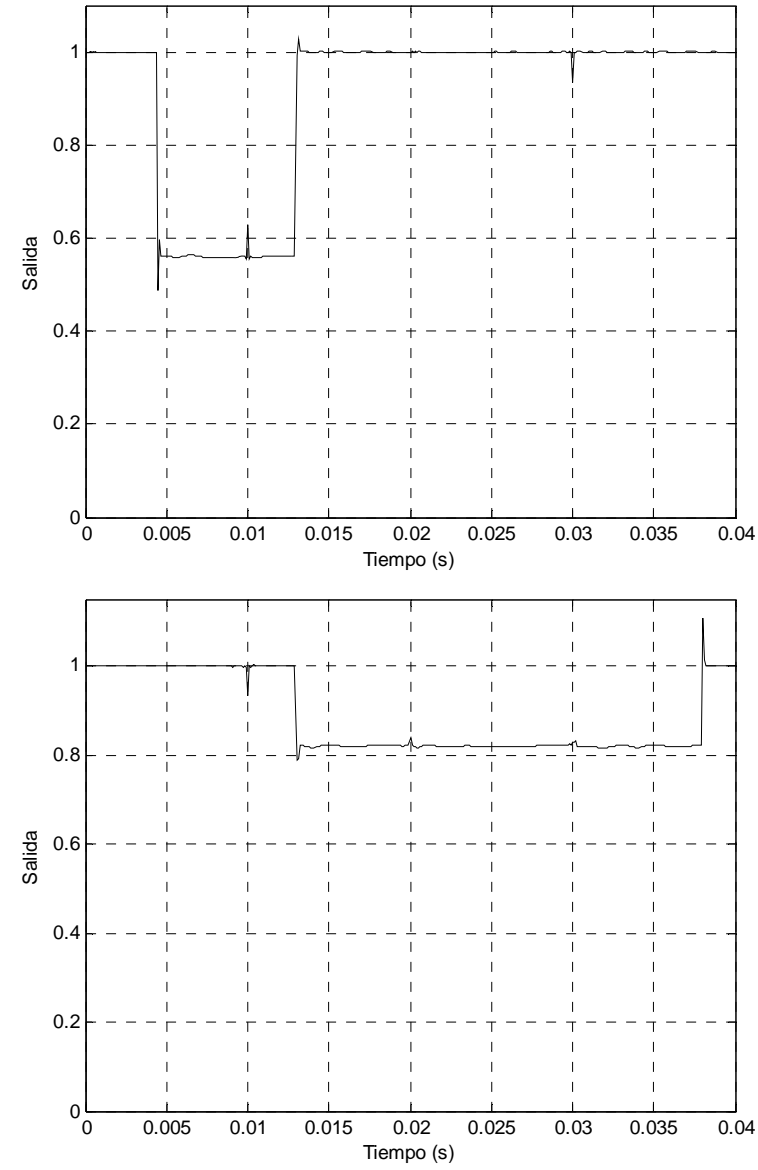

Fig. 8. Output of the network in the presence of $69 \%, 44 \%$ and $18 \%$ voltage sags

It can be appreciated that the network works in a suitable form, detecting the voltage sag, including the initial and final instant, and the duration of the sag.
The mean of the output results $s_{\text {med }}=0,31$ with an standard deviation of Std=0,048 for the firs sag, $\mathrm{s}_{\text {med }}=$ 0,56 and $S t d=0,049$ for the second sag and $s_{\text {med }}=0,82$ and $\operatorname{Std}=0,012$ for the third sag.

The three voltages inputs in $t, t-\Delta t$ and $t-2 \Delta t$ are needed to guarantee the dynamic response of the neural network. It is chosen a value of $\Delta t=0,1 \mathrm{~ms}$. It is possible, however, to improve the precision of the response if several sizes of the input/output data packages are tested.

\section{B. Application to an Electric Power System}

Finally, the performance of the neural network with the occurrence of a fault in an electric power system was tested. That power system is represented en figure 9 and it was simulated in Matlab/Simulink helped by SimPowerSystem blockset.

The system is constituted with a medium voltage source connected to a $20 \mathrm{kV} / 400 \mathrm{~V}$ transformer. It is supplying two loads through a distribution line. The first load is localized in the middle of the line, with the addition of a capacitor bank to improve de power factor. The second load is connected at the end of the line with another capacitor bank.

The three-phase fault occurs in the point of connection of the second load. The initial instant of the fault is 16,67 $\mathrm{ms}$ and the final instant is $133,3 \mathrm{~ms}$. The voltage $V_{R N}$, figure 10a, was measured at the point of connection of the first load. The neural network detected initial and final time and the magnitude of the voltage sag (figure 10.b).

In figure 10, the voltage at the measurement point and the output of neural network are presented in per unit values.

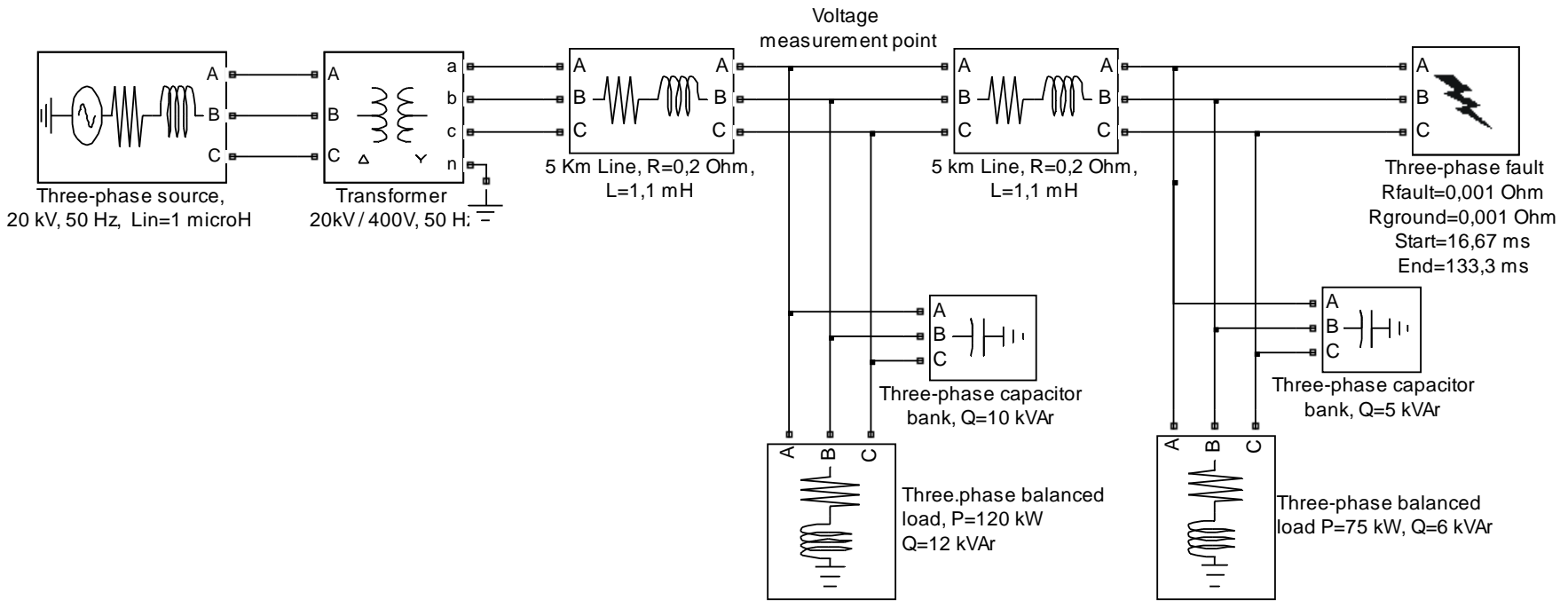

Fig. 9. Simulink scheme of the simulated electric power system 


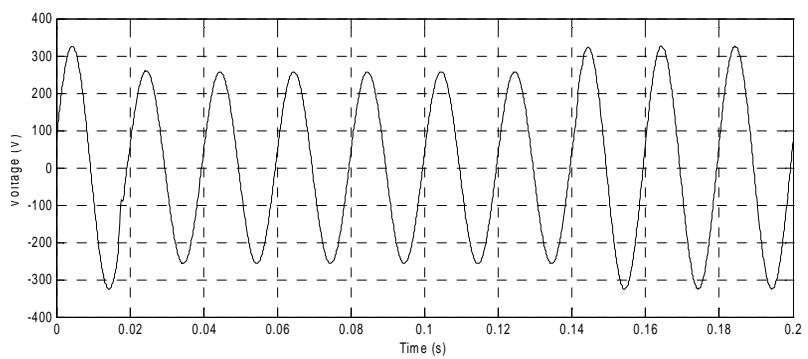

a)

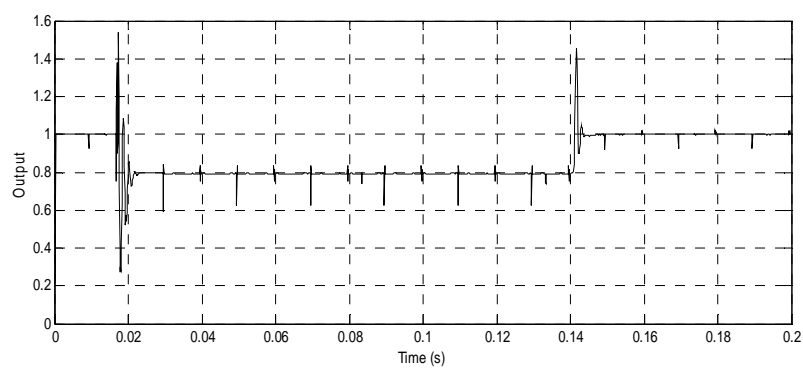

b)

Fig. 10. Output of the neural network (system per unit)

The measured voltage during the sag is $182,14 \mathrm{~V}$ and the answer of the neural network during the sag is $o=0,79$. The output of the network presents oscillations at the beginning and at the end of the sag. This is because of the neural networks had been trained to detect ideal sags, without the presence of transients, as those presented in these voltage waveform. The network performance can be improved if it is trained to detect the transient periods too.

\section{Conclusions}

A procedure to measure on-line voltage disturbances using artificial neural networks has been presented. The feedforward neural network designed has been trained with a backpropagation method, using input/output data supplied with computer simulations. The training data were introduced in incremental mode.
To have an adequate dynamic response of the disturbance detection system, three inputs were considered for the neural network, the voltage at instant $t$, and at two preceding instants, $t-\Delta t$ and $t-2 \Delta t$.

Finally, the neural network was satisfactorily tested for the detection and measurement of different voltage sags, including sags caused by the electrical system faults.

\section{References}

[1] "European Standard UNE-EN 50160: Voltage characteristics on public distribution grids". AENOR ,2001.

[2] "Estimating economic impact of voltage sags". Wang, J.; Chen, S.; Lie, T.T. International Conference on Power System Technology, 2004. Vol. 1, 21-24 Nov. Pp. 350-355.

[3] "Voltage sag vulnerability study in power system planning”. Wang, A.C.; Teng, J.H.; Shen, C.C.; Lu, C.N.; Chen, Y.T.; Huang, C.Y.; Liu, E.. IEEE Power Engineering Society General Meeting, 2005. Pp. 383-388.

[4] "Fast Estimation of Voltage and Current Phasors in Power Networks Using an Adaptive Neural Network”. P. K. Dash, S. K. Panda, D. P. Swain. IEEE Transactions on Power Systems, vol. 4, no. 12, 1997, pp. 1494-1499.

[5] "A new technique for unbalance current and voltage estimation with neural networks”. F. J. Alcántara, P. Salmerón. IEEE Trans. On Power Systems, Vol. 20, No. 2, 2005, pp. 852-858.

[6] "Power quality disturbance data compression using wavelet transforms methods”, S. Santoso, E.J. Powers, IEEE Trans. On Power delivery, Vol 12, No 3, July 1997. 